\title{
The relationship between neutrophil/lymphocyte ratio and uric acid levels in multiple sclerosis patients
}

\author{
Bolayir $\mathrm{A}^{1}$, Cigdem $\mathrm{B}^{1}$, Gokce $\mathrm{SF}^{1}$, Yilmaz $\mathrm{D}^{2}$ \\ Sivas Cumhuriyet University, Medicine Faculty, Neurology Department, Sivas, Turkey. \\ asliarslanturk@gmail.com
}

\begin{abstract}
BACKGROUND: In this study, we aimed to determine whether neutrophil / lymphocyte ratio (NLR), obtained by dividing the number of neutrophils by the number of lymphocytes, and uric acid (UA) levels in multiple sclerosis (MS) patients vary compared with healthy controls and to establish correlations among these changes themselves as well as between such changes and MS subtypes, immunomodulatory drug use, the duration of the disease and prognosis.

METHODS: 150 patients who presented to our hospital and were diagnosed with MS and 150 healthy volunteers were retrospectively included in our study. EDSS score (Expanded Disability Status Scale) was used to assess the disability of the patients.

RESULTS: Compared to healthy volunteers, MS patients had lower UA levels $(p<0.001)$ and higher

NLR values $(p=0.02)$. In addition, UA levels were higher in patients with a low EDSS score or those on immunomodulating drugs $(p<0.001, p=0.04$, respectively). NLR value was lower in patients with a low EDSS score $(p<0.001)$. There was a negative correlation between NLR value and UA $(r=-0.23, p=0.003)$. Similarly, UA level decreased with increasing EDSS score and duration of disease $(r=-0.38, p<0.001 ; r=$ $-0.17, p=0.02$, respectively).

CONCLUSION: Evaluating the NLR value, recognized as a new marker for inflammation in MS, together with the UA value, thought to be protective in MS, might be more effective than evaluating these parameters alone in demonstrating disability in patients (Tab. 4, Ref. 28). Text in PDF www.elis.sk KEY WORDS: neutrophil/lymphocyte ratio, uric acid, multiple sclerosis, inflammation, Expanded Disability Status Scale.
\end{abstract}

\section{Introduction}

Multiple sclerosis (MS), a chronic inflammatory demyelinating disease, is the disease of the central nervous system (CNS) most often associated with disability in young adults $(1,2)$. Its multifactorial etiology includes genetic and environmental risk factors. It has more than one subtype. Relapsing-remitting MS (RRMS), its most common subtype, is characterized by repetitive typical neurological symptoms that last several days to weeks, with a spontaneous recovery period or a recovery period in response to treatment with steroids. In its subtype, known as secondary progressive MS (SPMS), a patient has a CNS disorder that continues with a chronic process with or without relapses that increase disability. The primary progressive MS (PPMS) subtype with rapid deterioration in a short period of time from the onset of the disease is characterized by progressively deteriorating clinical course. In clinically isolated syndrome (CIS), another MS subtype, there are neurological signs

${ }^{1}$ Sivas Cumhuriyet University, Medicine Faculty, Neurology Department, Sivas, Turkey, and ${ }^{2}$ Nigde Omer Halisdemir University Training and Research Hospital, Neurology Department, Nigde, Turkey

Address for correspondence: A. Bolayir, Sivas Cumhuriyet University Neurology Department Sivas, Turkey.

Phone:+905423853005 and symptoms caused by inflammation and demyelination in CNS although MS diagnostic criteria are not fully met.

Systemic inflammation and autoimmunity, which can lead to chronic neurodegeneration, are assumed to play an important role in MS pathogenesis through the activation of both innate and adaptive immune cells and the production of proinflammatory cytokines that cause an inflammatory response in CNS $(3,4)$. Therefore, many easily accessible biomarkers that may be useful in diagnosing MS disease and predicting prognosis have been investigated (e.g. tumor necrosis factor-alpha (TNF- $\alpha$ ) and interleukin (IL) -6); however none of them have been proven to be clinically useful.

In addition, it has been observed that serum levels of uric acid (UA) (5-7), thought to play an important role in the development of neurodegeneration, are lower in MS patients (8). UA is a natural cleanser of peroxynitrite, a toxic product of nitric oxide and superoxide inducing inflammation, demyelination and axonal damage in MS pathogenesis (9). It has also been suggested that UA levels may be a clinical indicator of disease activity in MS patients (9).

The differential number of white blood cells is a commonly used biomarker to show systemic inflammation. Recent studies have shown that the neutrophil / lymphocyte ratio (NLR) is a better indicator of neutrophil or lymphocyte count alone in demonstrating systemic inflammation (10). Although it is known that there is a relationship between increased NLR and MS, the results regard- 


\section{$357-361$}

ing the relationship between MS-related disability and NLR have been inconsistent $(4,10,11)$.

We conducted this study to determine the changes in the NLR and UA levels of MS patients by comparing them with healthy controls and establish correlations among these changes themselves as well as between such changes and MS subtypes, medications used, the duration of the disease and prognosis.

\section{Materials and methods}

\section{Determination of study group}

In our study, patient records between January 2017 and January 2018 were retrospectively analyzed, and 150 patients who presented to our hospital between these dates and who were diagnosed with MS by an expert neurologist, and 150 age-andsex-matched healthy volunteers were included in our patient group. No limitation by age or sex was applied in the patient and control groups. $2010 \mathrm{McDonald}$ 's criteria were taken as the basis for diagnosis of MS. Patients in our patient group were in the remission period clinically and radiologically in the last 3 months before they were included in the study and did not receive steroid treatment. Determination of whether or not the patients were in clinical remission was based on past medical history and neurological examination of the patients, and the patients' most recent cranial and spinal magnetic resonance images (MRI) were used to determine radiological remission, and those without active contrast enhancement were considered radiologically in remission. EDSS score (Expanded Disability Status Scale) was used to assess the disability of the patients. The date when the patient was first diagnosed was taken as the basis to calculate the duration of the disease, and the treatments received by the patients were the therapies they were receiving at the time of collection of the blood samples. Patients included in our study received first-line immunomodulating therapy (interferons and glatiramer acetate), and patients who received other first or second-line immunomodulating therapy known to affect lymphocyte count were excluded from our study. The rights of all participants were protected according to the Helsinki Declaration. Patients under eighteen, those with active infection, diabetes mellitus, hypertension, acute / chronic liver or kidney failure, acand EDSS. tive or chronic inflammatory disease (e.g. inflammatory bowel disease, Sjögren's syndrome), other autoimmune disease, pregnancy, existing malignancy or those with a history of surgical intervention in the last 3 months, use of medications that may affect serum UA level, acute myocardial infarction or trauma were not included in our study.

Our control group comprised 150 age-and- sex-matched healthy volunteers without any systemic disease, who did not take any systemic medications and who gave blood for other reasons.

Tab. 1. The demographic characteristics of patient and control groups.

\begin{tabular}{lccc}
\hline & $\begin{array}{c}\text { Patients } \\
(\mathrm{n}=150)\end{array}$ & $\begin{array}{c}\text { Control } \\
(\mathrm{n}=150)\end{array}$ & $\mathrm{p}$ \\
\hline Sex (female) & $105(70 \%)$ & $110(73.3 \%)$ & 0.52 \\
\hline Age (years) & $37.01 \pm 7.56$ & $37.57 \pm 9.21$ & 0.67 \\
\hline Uric acid (mg/dL) & $3.94 \pm 1.11$ & $4.56 \pm 1.22$ & $<0.001$ \\
\hline NLR & $2.38 \pm 1.07$ & $2.02 \pm 0.82$ & 0.02 \\
\hline MS subtype & & & \\
CIS & $3(2 \%)$ & & \\
RRMS & $126(84 \%)$ & & \\
PPMS & $8(5.3 \%)$ & & \\
SPMS & $13(8.7 \%)$ & & \\
\hline Medications Used & & & \\
Interferon beta-1a (3 times a week) & $62(41.3 \%)$ & & \\
Interferon beta-1a (Once a week) & $19(12.7 \%)$ & & \\
Interferon beta-1b & $28(18.7 \%)$ & & \\
Glatiramer Acetate & $26(17.3 \%)$ & & \\
No treatment & $15(10 \%)$ & & \\
\hline Disease Duration (years) & $6.32 \pm 4.71$ & & \\
\hline EDSS & $2.87 \pm 1.85$ & & \\
\hline EDSS & & \\
3 and Below 3 & $95(63.3 \%)$ & & \\
Over 3 & $55(36.7 \%)$ & \\
\hline
\end{tabular}

NLR - neutrophil lymphocyte ratio, CIS - clinically isolated syndrome, MS - multiple sclerosis, RRMS - relapsing-remitting multiple sclerosis, PPMS - primary progressive multiple sclerosis, SPMS - secondary progressive multiple sclerosis, EDSS - Expanded Disability Status Scale

Tab. 2. Relationship of uric acid levels in the patient group to MS subtype, medication used

\begin{tabular}{lccc}
\hline & $\begin{array}{c}\text { Uric acid } \\
(\mathrm{mg} / \mathrm{dL})\end{array}$ & $\mathrm{p}^{1}$ value & $\mathrm{p}^{2}$ value \\
\hline MS subtype & & & \\
$\quad$ CIS & $3.80 \pm 1.99$ & 0.33 & \\
RRMS & $4.00 \pm 1.09$ & & \\
PPMS & $3.81 \pm 1.58$ & & \\
SPMS & $3.50 \pm 0.81$ & & $0.001^{a}$ \\
\hline Medications Used & & 0.04 & $0.001^{b}$ \\
$\quad$ Interferon beta-1a (3 times a week) & $4.16 \pm 1.13$ & & $0.006^{c}$ \\
$\quad$ Interferon beta-1a (Once a week) & $4.22 \pm 1.04$ & & \\
Interferon beta-1b & $3.65 \pm 1.12$ & & \\
$\quad$ Glatiramer Acetate & $4.04 \pm 1.04$ & & \\
$\quad$ No treatment & $3.07 \pm 0.79$ & & \\
EDSS & & & \\
$\quad$ 3 and Below 3 & $4.28 \pm 1.10$ & & \\
Over 3 & $3.36 \pm 0.87$ & & \\
\hline
\end{tabular}

MS - multiple sclerosis, CIS - clinically isolated syndrome, RRMS - relapsing-remitting multiple sclerosis, PPMS - primary progressive multiple sclerosis, SPMS - secondary progressive multiple sclerosis, EDSS - Expanded Disability Status Scale. ${ }^{a}-$ Interferon beta-1a ( 3 times a week) - No treatment $^{\mathrm{b}}-$ Interferon beta-1a (Once a week) - No treatment, ${ }^{\mathrm{c}}-$ Interferon beta- $1 \mathrm{~b}-$ No treatment, ${ }^{\mathrm{d}}-$ Glatiramer Acetate - No treatment 
Tab. 3. Relationship of NLR levels in the patient group to MS subtype, medication used and EDSS.

\begin{tabular}{lcc}
\hline & \multicolumn{1}{c}{ NLR } & $\mathrm{p}$ \\
\hline MS subtype & $1.62 \pm 0.32$ & 0.308 \\
CIS & $2.37 \pm 1.08$ & \\
RRMS & $2.28 \pm 0.78$ & \\
PPMS & $2.72 \pm 1.16$ & \\
SPMS & & \\
\hline Medications Used & $2.40 \pm 1.2$ & \\
Interferon beta-1a (3 times a week) & $2.36 \pm 1.0$ & \\
Interferon beta-1a (Once a week) & $2.41 \pm 1.11$ & \\
Interferon beta-1b & $2.30 \pm 0.87$ & \\
Glatiramer Acetate & $2.38 \pm 0.90$ & \\
No treatment & & \\
\hline EDSS & $2.13 \pm 0.94$ \\
3 and Below 3 & $2.81 \pm 1.14$ \\
Over 3 & \\
\hline \multicolumn{2}{l}{ NLR - neutrophil lymphocyte ratio, MS - multiple sclerosis, CIS - clinically iso- } \\
lated syndrome, RRMS - relapsing-remitting multiple sclerosis, PPMS - primary \\
progressive multiple sclerosis, SPMS - secondary progressive multiple sclerosis, \\
EDSS - Expanded Disability Status Scale
\end{tabular}

Ethics committee approval of our study was obtained from Sivas Cumhuriyet University Ethics Committee and our ethics committee approval number was 2018-03/12.

\section{Assessment of biochemical and hematological parameters}

Dry tubes were used for biochemical analysis and EDTA tubes for hematological tests. While the complete blood count was analyzed using the Diagon kits on the Mindray BC-6800 device, biochemical analyses were conducted by fully automated nephelometric method on Beckman Coulter AU5800(Beckman Coulter Inc, Hialeah, Florida) using the kits of the same company. NLR was calculated by dividing the neutrophil count by lymphocyte count obtained from the complete blood count.

\section{Statistical method}

The data obtained from our study were uploaded to SPSS (Ver: 22.0) software package and evaluated. Whether the distribution of continuous data is normal or not was examined using the Kolmogorov-Smirnov test. Continuous data were described as mean \pm standard deviation (SD) when they showed a distribution close to normal, otherwise as median and interquartile range, while categorical data were expressed as frequency and percentage. The chi-square test was used to compare ratios, where appropriate, while Student's t test was utilized to check continuous variables. Kruskal-Wallis H test was used to compare the values of three independent groups. The Spearman's rank-order correlation test was employed to establish whether there's any statistically significant relationship between quantitative variables. Values of $p$ $<0.05$ were considered significant.

\section{Results}

While the percentage of female was statistically higher in both patient and control groups, the percentage of male was statistically lower, and there was no significant difference in sex distribution between the two groups $(p=0.52)$. Mean age of the patient group was $37.01 \pm 7.56$ and that of the control group was $37.57 \pm 9.21(p=0.67)$. Mean UA value in the patient group (3.94 \pm 1.11 ) was statistically significantly lower than the control group $(p<0.001)$, whereas NLR value was statistically significantly higher $(\mathrm{p}=0.02)$.

The majority of MS patients in the patient group $(84 \%, n=126)$ were RRMS patients, with the rest comprising 13 SPMS patients (8.7\%), 8 PPMS (5.3 \%) patients and 3 (2\%) CIS patients. When evaluating medication use, patients were mostly on interferon beta1a $(54 \%)$. Mean duration of the disease was $6.32 \pm 4.71$ years. In addition, while the mean EDSS value of the patients was 2.87 \pm 1.85 , the EDSS values of most patients were 3 and below 3 (63.3\%) (Tab. 1).

When the patient group was evaluated in terms of UA levels, there was no statistically significant difference in UA levels by MS subtypes $(p=0.33)$, whereas there was a statistically significant difference by medications used and the fact that the EDSS value is 3 and below or above 3 ( $p=0.04, p<0.001$, respectively). In the assessment made in terms of medications used, using any of the first-line immunomodulating medications (interferons and glatiramer acetate) in MS treatment statistically significantly increased the level of UA. Similarly, the UA level was statistically significantly higher in patients with an EDSS value of 3 or less $(\mathrm{p}<0.001)$ (Tab. 2).

Similarly, when the patient group was evaluated in terms of NLR values, there was no statistically significant difference in NLR value by MS subtypes and medications used $(\mathrm{p}=0.308, \mathrm{p}$ $=0.99$, respectively), whereas the NLR value was statistically significantly lower in patients with an EDSS value of 3 or less ( $p$ $<0.001$ ) (Tab. 3).

No statistically significant correlation was found between UA levels and age in the patient group, and UA level increased with decreasing NLR, EDSS score and disease duration $(\mathrm{r}=-0.23$ and $\mathrm{p}=$ $0.003 ; \mathrm{r}=-0.38$ and $\mathrm{p}<0.001 ; \mathrm{r}=-0.17$ and $\mathrm{p}=0.020$, respectively). With NLR values, no statistically significant correlation was noted between age and disease duration ( $\mathrm{p}$ $>0.05$ ), whereas NLR values increased statistically significantly with increasing EDSS score $(r=0.23$ and $\mathrm{p}=0.003$, respectively) (Tab. 4). In addition, UA levels in the control group increased with increasing age $(\mathrm{r}$ $=-0.21$ and $\mathrm{p}=0.005)$.
Tab. 4. Correlation coefficients between age, EDSS sco
NLR levels in the patient group and significance levels.

\begin{tabular}{lccccc}
\hline & \multicolumn{2}{c}{ Uric acid } & & & \multicolumn{2}{c}{ NLR } \\
\cline { 2 - 3 } \cline { 5 - 6 } & $\begin{array}{c}\text { Correlation } \\
\text { coefficient }\end{array}$ & $\mathrm{p}$ & & $\begin{array}{c}\text { Correlation } \\
\text { coefficient }\end{array}$ & $\mathrm{p}$ \\
\hline Age & -0.10 & 0.11 & & 0.04 & 0.32 \\
EDSS & -0.38 & $<0.001$ & & 0.23 & 0.003 \\
Duration of disease & -0.17 & 0.020 & & 0.02 & 0.43 \\
NLR & -0.23 & 0.003 & & - & - \\
\hline
\end{tabular}

NLR - neutrophil lymphocyte ratio, EDSS - Expanded Disability Status Scale 


\section{Discussion}

Based on the results of our study, we can say that compared to healthy volunteers, MS patients had lower UA levels and higher NLR values. In addition, UA levels were not affected by the MS subtype but were higher in patients with a low EDSS score or those on first-line immunomodulating medications (interferons and glatiramer acetate). Besides, the NLR value unaffected by either the MS subtype or use of first-line immunomodulating medications (interferons and glatiramer acetate) was lower in patients with a low EDSS score. There is also a negative relationship between NLR value and UA. Similarly, UA level decreased with increasing EDSS score and duration of disease.

MS, as is known, is a disease in which inflammation and neurodegeneration play a role, contributing to neuronal demyelination and axonal injury (1). Reactive oxygen and nitrogen derivatives are known to play an active role in this inflammatory process (12). In both experimental allergic encephalomyelitis, which is an MS model, and in MS, inflammatory cells produce oxidizing derivatives such as nitric oxide and peroxynitrite (13). It is thought that peroxynitrite, a toxic product of free radicals of nitric oxide and superoxide, can play an important role in the pathophysiology of MS (14). Therefore, it has been suggested that UA, a natural peroxynitrite cleaner and endogenous antioxidant, may have a protective effect in MS patients. In MS animal models, the exogenous administration of UA has been shown to prevent tissue damage in the CNS and clinical manifestations of the disease (15, 16). There are also some studies showing that MS disease may be associated with lower serum levels of UA and that UA can be used as a preclinical biomarker in MS patients. Epidemiological studies have demonstrated that people with gout disease, characterized by increased UA concentrations in serum, are less likely to have MS disease compared to the population (14). In addition, the results of another study in which more than 20 million patient records were evaluated statistically showed that hyperuricemia can be protective against MS (17). In our study, similar to these results, UA values were low in MS patients regardless of MS subtype. In addition, an inverse relationship was identified between UA levels and EDSS values, which concurs well with $(18,19)$. Similar to Li et al.'s study, our study showed that patients with an EDSS value of 3 or less had higher UA levels compared to other patients. This result can be ascribed to the fact that high UA levels play a protective role, being associated with less disability in MS disease. Furthermore, there is a negative linear correlation between UA levels and disease duration, in other words, UA levels decrease with increasing disease duration. Based on this result, it can be said that protective mechanisms are more active in the early stages of the disease and lose their effects over time, and disability increases with the resulting degenerative process. Similarly, UA levels in patients on any of the first-line immunomodulating medications (interferons and glatiramer acetate) were higher than those not taking any medication. These results suggest that taking any first-line immunomodulating medication (interferons and glatiramer acetate) improves prognosis by reducing development of disability associated with MS.
There are many studies investigating the levels of inflammatory markers in MS patients $(20,21)$. A study by Martins et al. claimed that the increase in the level of proinflammatory cytokines, including interferon (IFN) $-\gamma$, IL-2, IL-1, TNF- $\alpha$, IL-4, IL-10 and IL-13, has an effect on disease progression (20). Moreover, proinflammatory $\mathrm{T}$ helper 1 cells are known to contribute to the development of new lesions, while T helper 2 cells suppress local inflammation (22). NLR, thought to be a good indicator of showing systemic inflammation and obtained by dividing the neutrophil count by the lymphocyte count, has been shown to be an important biomarker in autoimmune diseases such as rheumatoid arthritis and inflammatory bowel disease $(23,24)$. High NLR values are recognized as a prognostic indicator in many diseases including acute ischemic stroke, myocardial infarction and gastrointestinal tract malignancies (25-27). While the relationship of NLR with MS has been shown in many studies (4), its correlation with MSrelated disability is unclear (10-11). Similar to the results of aforementioned studies, we found higher NLR values in MS patients, regardless of MS subtype, compared to the controls. Our results are not surprising for MS disease, in which inflammatory processes are known to play an active role in its pathophysiology, and support the view that the inflammatory response of CNS in MS also depends on the peripheral immune system (28). Besides, according to the results of our study, using any of the first-line immunomodulating medications (interferons and glatiramer acetate) has no significant effect on NLR value, which suggests that this may be the underlying cause of more limited anti-inflammatory efficacy of these immunomodulating medications used to treat MS. Likewise, in agreement with previous studies (4), we found that patients with an EDSS value higher than 3 had higher NLR values compared to other patients, with a linear correlation established between NLR value and EDSS, which implies that evaluating the inflammatory level of patients can help determine the severity of MS disease.

Furthermore, our study showed that there's an inverse correlation between NLR value, an inflammatory marker, and UA level, which is thought to have a protective role in MS disease, so, NLR levels decrease with increasing UA levels. Our findings would seem to show that evaluating the NLR values (shown to be associated with poor prognosis and disability in all MS subtypes) together with UA levels (known to have a protective role) is more useful in gaining a better insight into the future prognosis of the disease. From this point of view, our study was the first study to evaluate NLR and UA values together in MS patients versus healthy volunteers. Additionally, these two parameters can be easily studied in peripheral blood without additional costs or intervention, which makes these two biomarkers more beneficial.

Our study has several limitations. First, our study was a retrospective study and therefore we were able to include only the patients whose records were available in our hospital's automation system in the study. Otherwise, since the number of patients was low, the number of SPMS and PPMS patients remained relatively low and therefore our statistical data on the subgroups were weak. Furthermore, we studied only the NLR values (one of inflammatory indicators) and UA levels in our patients. Therefore, other inflammatory indicators and the levels of other intermediate and residual 
products were not evaluated. Withal, the patients' clinical conditions were determined only by EDSS values, and we were unable to apply other more specific and sensitive MS scales. Lastly, only active contrast-enhancing lesions on cranial and spinal MR images were considered in our study. Thus, other radiological changes such as development of a new T2 lesion or cortical atrophy were not included in the study, and the relationship between such changes and NLR and UA levels were not revealed.

\section{Conclusions}

The study demonstrated that MS patients had higher NLR values and lower UA levels. Moreover, a positive correlation was identified between NLR values and EDSS, whereas a negative correlation was determined between UA levels and EDSS, disease duration and NLR. Besides, both low UA levels and high NLR values are associated with disability in MS patients. These results have led us to conclude that evaluating the NLR value, recognized as a new marker for inflammation in MS, together with the UA value, might be more effective than evaluating these parameters alone in demonstrating disability in MS patients. Prospective randomized controlled trials with greater detail and a higher number of patients are required to be able to make further comments.

\section{References}

1. Ebers GC. Environmental factors and multiple sclerosis. Lancet Neurol 2008; 7 (3): 268-277 .

2. Zostawa J, Adamczyk J, Sowa $\mathbf{P}$ et al. The influence of sodium on pathophysiology of multiple sclerosis. Neurol Sci 2017; 38 (3): 389-398.

3. Perry VH, Cunningham C, Holmes C. Systemic infections and inflammation affect chronic neurodegeneration. Nat Rev Immunol 2007; 7: $161-167$.

4. Demirci S, Demirci S, Kutluhan S et al. The clinical significance of the neutrophil-to-lymphocyte ratio in multiple sclerosis. International Journal of Neuroscience 2016; 126 (8): 700-706.

5. Liu B, Shen Y, Xiao K et al. Serum uric acid levels in patients with multiple sclerosis: a meta-analysis. Neurol Res 2012; 34 (2): 163-171.

6. Wang $\mathrm{L}, \mathrm{Hu} \mathrm{W}$, Wang $\mathrm{J}$ et al. Low serum uric acid levels in patients with multiple sclerosis and neuromyelitis optica: an updated meta-analysis. Mult Scler Relat Disord 2016; 9: 17-22.

7. Guerrero AL, Gutiérrez F, Iglesias F et al. Serum uric acid levels in multiple sclerosis patients inversely correlate with disability. Neurol Sci 2011; 32 (2): 347-350.

8. Maesaka JK, Wolf-Klein G, Piccione JM et al. Hypouricemia, abnormal renal tubular urate transport, and plasma natriuretic factor (s) in patients with Alzheimer's disease. J Am Geriatr Soc 1993; 41 (5): 501-506.

9. Toncev G, Milicic B, Toncev S et al. Serum uric acid levels in multiple sclerosis patients correlate with activity of disease and blood-brain barrier dysfunction. Eur J Neurol 2002; 9 (3): 221-226.

10. Guzel I, Mungan S, Oztekin ZN et al. Is there an association between the Expanded Disability Status Scale and inflammatory markers in multiple sclerosis? J Chin Med Assoc 2016; 79: 54-57.
11. Bisgaard AK, Pihl-Jensen G, Frederiksen JL. The neutrophil-tolymphocyte ratio as disease activity marker in multiple sclerosis and optic neuritis. Multi Scler Relat Dis 2017; 18: 213-217

12. Smith KJ, Kapoor R, Felts PA. Demyelination: the role of reactive oxygen and nitrogen species. Brain Pathol 1999; 9: 69- 92

13. Mattle HP, Lienert C, Greeve I. Uric acid and multiple sclerosis. Ther Umsch 2004; 61: 553-555.

14. Hooper DC, Scott GS, Zborek A et al. Uric acid, a peroxynitrite scavenger, inhibits CNS inflammation, blood-CNS barrier permeability changes, and tissue damage in a mouse model of multiple sclerosis. FASEB J 2000; 14: 691-698.

15. Hooper DC, Spitsin S, Kean RB et al. Uric acid, a natural scavenger of peroxynitrite, in experimental allergic encephalomyelitis and multiple sclerosis. Proc Natl Acad Sci 1998; 95: 675-680.

16. Kean RB, Spitsin SV, Mikheeva $T$ et al. The peroxynitrite scavenger uric acid prevents inflammatory cell invasion into the central nervous system in experimental allergic encephalomyelitis through maintenance of blood- central nervous system barrier integrity. J Immunol 2000; 165 : 6511-6518.

17. Staub M. Uric acid as a scavenger in oxidative stress. Orv Hetil 1999; 140: 275-279.

18. Rumbach L. EDSS expanded disability status scale. Rev Neurol 2000; 156: $1036-1040$.

19. Li J. Study on the relationship between serum uric acid levels and multiple sclerosis. Med Innov Res 2008; 5: 3.

20. Martins TB, Rose JW, Jaskowski TD et al. Analysis of proinflammatory and antiinflammatory cytokine serum concentrations in patients with multiple sclerosis by using a multiplexed immunoassay. Am J Clin Pathol 2011; 136 (5): 696-704.

21. Uysal S, Meric Yilmaz F, Bogdaycioglu $\mathbf{N}$ et al. Increased serum levels of some inflammatory markers in patients with multiple sclerosis. Minerva Medica 2014; 105 (3): 229-235

22. Ziaber J, Pasnik J, Baj Z et al. The immunoregulatory abilities of polymorphonuclear neutrophils in the course of multiple sclerosis. Mediat Inflamm 1998; 7: 335-338.

23. Fu H, Qin B, Hu Z et al. Neutrophil- and platelet-tolymphocyte ratios are correlated with disease activity in rheumatoid arthritis. Clin Lab 2015; 61: 269-273.

24. Acarturk G, Acay A, Demir K et al. Neutrophil-tolymphocyte ratio in inflammatory bowel disease - as a new predictor of disease severity. Bratisl Lek Listy 2015; 116: 213-217

25. Tokgoz S, Kayrak M, Akpinar Z et al. Neutrophil lymphocyte ratio as a predictor of stroke. J Stroke Cerebrovasc Dis 2013; 22: 1169-1174.

26. Arbel Y, Finkelstein A, Halkin A et al. Neutrophil/lymphocyte ratio is related to the severity of coronary artery disease and clinical outcome in patients undergoing angiography. Atherosclerosis 2012; 225: 456-460.

27. Ertas G, Sönmez O, Turfan M et al. Neutrophil/lymphocyte ratio is associated with thromboembolic stroke in patients with non-valvular atrial fibrillation. Journal of the neurological sciences 2013; 324 (1-2): 49-52.

28. Noseworthy JH, Luchinetti C, Rodriguez M et al. Multiple sclerosis. N Engl J Med 2000; 343: 938-952.

Received October 26, 2020. Accepted October 30, 2020. 